\title{
Study on the use of polypropylene meshes for the correction of abdominal parietal defects during abdominoplasty
}

\author{
CATALIN GHEORGHE BEJINARIU ${ }^{1 *}$, SIRAMONA POPESCU ${ }^{1}$, \\ SERBAN DRAGOSLOVEANU ${ }^{2}$, CARMEN GIUGLEA ${ }^{3}$, \\ SILVIU ADRIAN MARINESC ${ }^{1}$ \\ ${ }^{1}$ Bagdasar-Arseni Emergency Clinical Hospital, Department of Plastic and Reconstructive Surgery, 12 Şoseaua Berceni, \\ 041915, Bucharest, Romania \\ ${ }^{2}$ Foisor Hospital, 35-37 Ferdinand Blvd., 020132, Bucharest, Romania \\ 3 "Saint John" Emergency Clinical Hospital, Department of Plastic and Reconstructive Surgery, 13 Șoseaua Vitan-Barzești, \\ 042122, Bucharest, Romania
}

Abstract. The current paper presents the advantages and disadvantages of the polypropylene mesh used for patients who underwent abdominoplasty associated with the treatment of abdominal wall defects. The study was based on data obtained from 64 patients who benefited from abdominoplasty associated with the surgical treatment of abdominal parietal defects, such as: hernias, eventrations and diastasis. The results of the research show that the use of the polypropylene mesh is associated with an increased resistance of the rectus abdominis sheath plication, the reduction of the postoperative complications rate and the improvement of the patients' satisfaction regarding the aesthetic result of the surgical intervention.

Keywords: abdominoplasty, polypropylene mesh, hernia treatment, diastasis treatment

\section{Introduction}

The chemical and physical properties of polypropylene meshes have been extensively studied in the international scientific community, with chemical stability, high resistance and high safety profile being just a few of the features that have significantly increased the popularity of these devices worldwide (Scheme 1) [1].

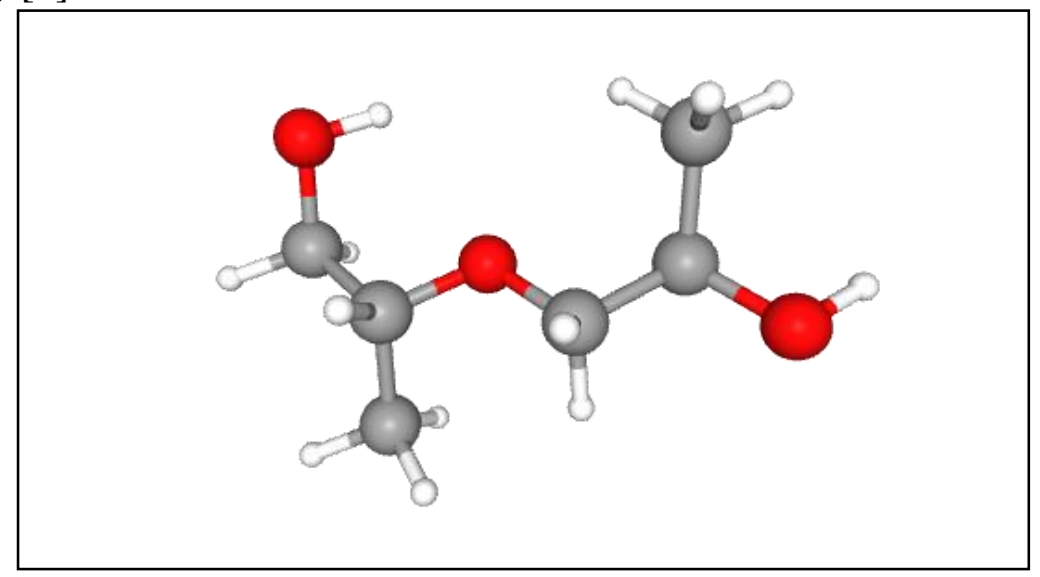

Scheme 1. Polypropylene $\left(\mathrm{C}_{6} \mathrm{H}_{14} \mathrm{O}_{3}\right)$ 3D graphic representation [2]

It is well known that the use of polypropylene meshes significantly reduces the risk of the recurrence of hernias and eventrations by increasing parietal resistance [3]; the same physical principles contribute to maintaining the shape obtained after performing the abdominoplasty associated with the treatment of diastasis. Without a doubt, the use of polypropylene meshes in the classic 
abdominoplasty protocol is a matter of debate, there are many studies that state that this approach is not recommended and that these devices should be used only for complicated cases [4].

The specialized literature shows that the introduction of polypropylene meshes has revolutionized the treatment of abdominal parietal defects by drastically reducing the number of relapses [5]. Studies dedicated to the safety profile of these devices show that ideally the characteristics of the meshes used for strengthening the abdominal wall should include: biochemical stability, lack of allergenic character, lack of carcinogenic substances, capacity for sterilization and modelling, high physical resistance and increased flexibility [6]. Polypropylene has proven to be the compound that meets these criteria, given its two-dimensional chemical structure with increased elasticity, thus coping with the large amplitudes of motion that are characteristic of this anatomical region [7-8].

Based on this data, the study aims to present in an objective way the main advantages and disadvantages of using polypropylene meshes in the field of plastic and aesthetic surgery.

\section{Material and method}

In order to perform the study, the following inclusion criteria were developed and respected:

- the age of the patients was between 18 and 65 years;

- cutaneous and adipose tissue excess in the abdominal region with the formation of the characteristic aspect of abdominal apron;

- identification of the cutaneous excess in association with diastasis of the rectus abdominis muscles, abdominal hernia or eventration;

The exclusion criteria taken into account were:

- patients under the age of 18 or over 65;

- personal pathological history incompatible with the surgery (severe heart failure, renal failure, severe lung disease);

- psychic disorders with impact on the patient's discernment;

- unfavorable psychological context.

In order to appreciate the results, the following data were analyzed and interpreted: age, weight, metabolic profile, body mass index, personal pathological history, type of procedure, rate of postoperative complications, rate of hernia or eventration relapses, length of hospitalization time, degree of satisfaction related to the postoperative outcome and the probability of recommending this surgical procedure to other patients.

The patients' degree of satisfaction related to the postoperative outcome and the probability of recommending the surgery were appreciated using a scale of values with 10 units, 1 being the minimum value and 10 maximum value.

Regarding the surgical approach, the therapeutic conduct was influenced by the particularities of the cases, the necessity of parietal reinforcement being correlated with the patient's expectation related to the postoperative result (Figure 1). 


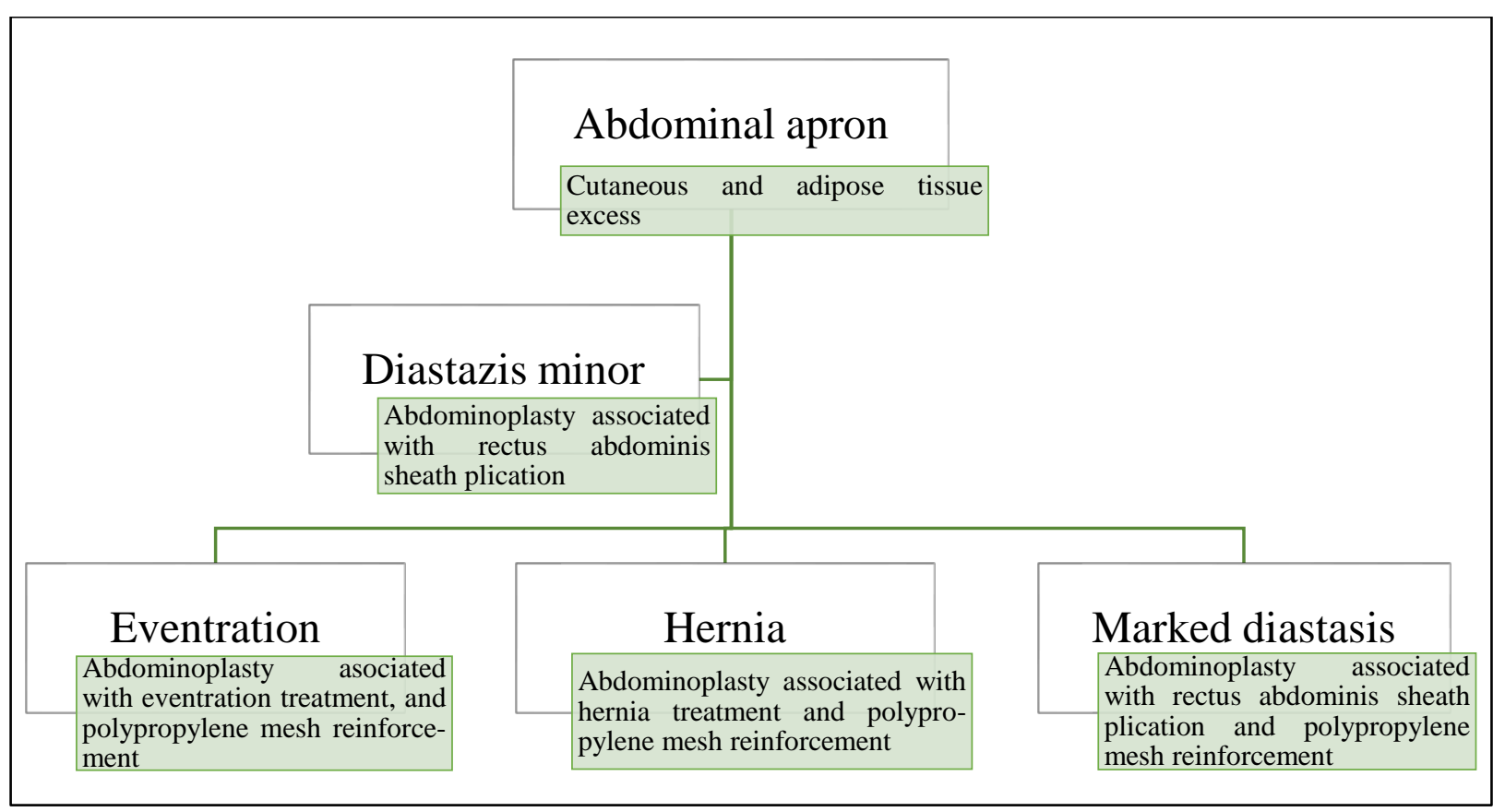

Figure 1. Therapeutic conduct related to the particularities of the case

\section{Results and discussions}

Following the statistical analysis, the average age of the patients who received surgical treatment was $42 \pm 3$ years, the age limits being 18 and 60 years, respectively. $79.68 \%$ (51 patients) of the patients came from urban areas.

The average value of the patients' body mass index included in the study was $26.2 \mathrm{~kg} / \mathrm{m}^{2}$, with the minimum value of $19.3 \mathrm{~kg} / \mathrm{m}^{2}$ and the maximum value of $29.8 \mathrm{~kg} / \mathrm{m}^{2}$. Regarding the fluctuations in weight, $73.43 \%$ of the patients included in the study had fluctuating weight, being overweight or obese for a period of time, followed by weight loss of at least $10 \mathrm{~kg}$.

Significant personal pathological history was observed in $54.68 \%$ of patients, 22 patients being diagnosed with hernia, 11 with eventration and 2 patients with type II diabetes. From the general group of patients, 5 patients had history of cardiovascular pathology: 3 patient with eventration, 1 patient diagnosed with umbilical hernia and 1 diabetic patient.

The type of surgical procedure was essentially influenced by the diagnosis and the local particularities of the patients, as follows:

1. For the 22 patients with abdominal cutaneous excess associated with hernia, the type of surgery was decided in a multidisciplinary team (plastic surgeon and general surgeon) and the operative protocol included excision of the cutaneous excess, treatment of the hernia followed by the rectus abdominis sheath plication and reinforcement with polypropylene mesh (Figure 2);
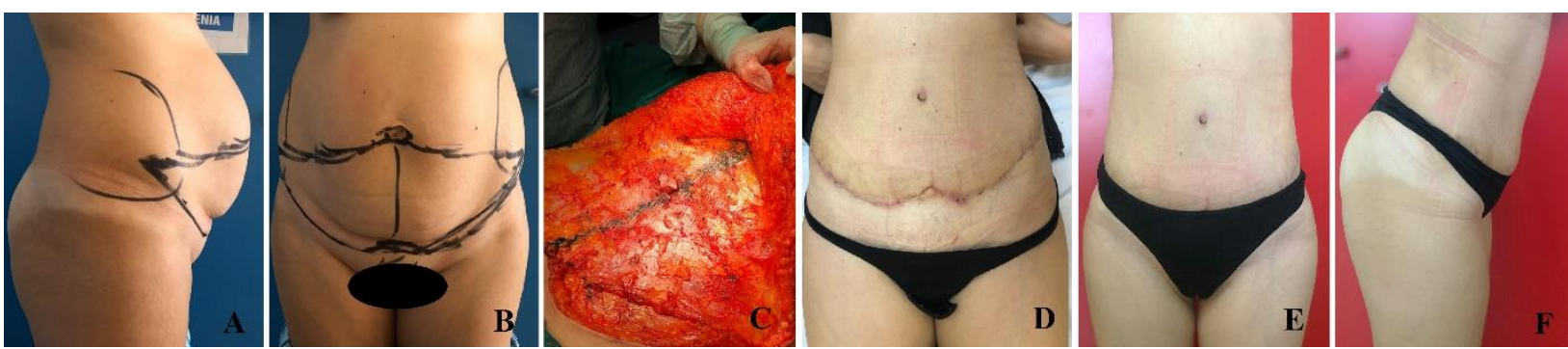

Figure 2. Surgical approach in case of a 35 years old patient with abdominal apron associated with umbilical hernia and marked diastasis - A. Preoperative lateral aspect; B. Preoperative front aspect; C. Polypropylene mesh in place; D. Abdominal scar 21 days after the surgery; E. Front view 30 days after the surgery; F. Lateral view 30 days after the surgery 
2. For the group of patients diagnosed with eventration involving 11 persons that have benefited from cutaneous excision, followed by the treatment of the eventration and the strengthening of the abdominal wall with polypropylene mesh;

3. For the group of patients who associated the cutaneous excess with the marked diastasis of the right abdominal muscles that included 25 persons who benefited from abdominoplasty, followed by rectus abdominis sheath plication and the parietal reinforcement with the polypropylene mesh;

4. For the group of patients with diastasis minor who underwent abdominoplasty, the classical protocol being applied for 6 persons, which involved the excision of the soft tissue excess associated with the rectus abdominis sheath plication.

Regarding the postoperative complications associated with these surgical procedures, they can be divided into two broad categories: complications of the tummy tuck, and complications of the parietal strengthening process. In the general group, postoperative complications were identified in 9 patients, 4 of them being specific for abdominoplasty and 5 being associated with complex parietal defects. The postoperative complications were relapse of the hernia (3 cases), persistence of diastasis ( 2 patients), dehiscence of the postoperative wound ( 2 patients), necrosis and seroma being identified in 1 patient.

The average length of hospitalization for the whole group was 2.4 days, with the limits between 24 hours and 5 days, this parameter being significantly influenced by the cases that have benefited from surgical reintervention for the treatment of relapses and complications.

The patient satisfaction regarding the postoperative aspect had an average value of 9.3 , being strongly influenced by the complication rate and the duration of the convalescent period. Regarding the probability of recommending this surgical procedure to other patients, the average value of this parameter was 9.1.

The polypropylene meshes, especially the light weight ones, offer benefits in terms of increasing the resistance of the abdominal wall, while maintaining the mobility and flexibility of the muscles. The pore structure allows for rapid and qualitative integration, increasing adherence to neighboring anatomical structures [9]. The mesh architecture allows the distribution of tension and forces throughout their surface, thus increasing the overall resistance of these devices. The physical and chemical properties of polypropylene meshes demonstrate their usefulness within a very diverse range of reconstructive surgeries, with alloplastic breast reconstruction and TRAM flap procedures being just a few examples in this regard [10-13].

The applicability of these devices in the case of patients with diastasis of the rectus abdominis muscles is supported on the one hand by the physical characteristics of these devices, and on the other hand by obtaining favorable and long-lasting aesthetic results. It should be mentioned that the maximum benefits are obtained for that category of patients whose diastasis persists under the firm contraction of the abdominal muscles, with the accentuation of the epigastric, hypogastric or global defect. In the case of patients whose appearance normalizes during the contraction of the abdominal muscles, the simple rectus abdominis sheath plication represents the optimal solution.

The literature shows that the use of the synthetic mesh in selected cases offers a considerable financial advantage, compared to the use of the acellular dermal matrix, in the conditions of a similar safety profile [14-16]. Recent research shows that the synthetic mesh may represent an alternative to the use of the acellular dermal matrix, but it remains a topic of debate [17].

Without a doubt, there may be some limitations regarding the use of these devices, in this sense, in the specialized literature, multiple cases are described that presents a possible association of synthetic meshes with the formation of postoperative seromas. The present study did not indicate an increase in the number of such complications, but the analysis of larger population groups could provide additional information.

Another element that must be analyzed is related to the development of prosthetic infections, the association of this type of complication requiring the extraction of the material as a part of the standard therapeutic protocol for all the surgical interventions that include the use of prosthetic materials. 
Regarding the legislative framework and the ethical norms, it should be mentioned that performing this kind of complex surgical interventions that involve the treatment of hernias and applying the meshes during the abdominoplasty, requires the involvement of a multidisciplinary surgical team including a plastic surgeon and a general surgeon. This approach allows to increase the safety profile of the surgery, reducing the risk of recurrence related to the parietal defect and obtaining favorable aesthetic results.

\section{Conclusions}

The results of the presented study support the fact that the use of polypropylene meshes in patients who benefit from abdominoplasty associated with the surgical treatment of abdominal parietal defects contributes to the increased resistance of the rectus abdominis sheath plication and to the reduction of the recurrence rate related to hernias and injuries.

The introduction of polypropylene meshes in the abdominoplasty protocol in patients with diastasis of the rectus abdominis muscles is associated with long-term maintenance of the desired shape and with an increased safety profile, sustained by the low rate of postoperative complications.

In this study, the use of polypropylene meshes was associated with an increased degree of patient satisfaction related to the postoperative outcome, as well as with an increased probability of recommending this surgical procedure to other patients.

\section{References}

1.AGARWAL, B.B., AGARWAL, K.A., MAHAJAN, K.C., Surg.Endosc ., 23, 2009, p. 242

2.***U.S. NATIONAL LIBRARY OF MEDICINE, [online] https://pubchem.ncbi.nlm.nih.gov/ compound/32881 [Accessed 02.11.2019].

3.SHIRAH, B.H., SHIRAH, H.A., J Med Imp Surg., no. 1, 2016, p. 1

4.TAVASSOLI, J., HAIAVY, J., The American Journal of Cosmetic Surgery, 28, no. 1, 2011, p. 37

5.KO, J.H., SALVAY, D.M., PAUL, B.C., WANG, E.C., DUMANIAN, G.A.,) Plast. Reconstr.Surg., 124, 2009, p. 836

6.AUFENACKER, T.J., KOELEMAY, M.J., GOUMA, D.J., SIMONS, M.P., Br. J. Surg., 93, 2006, p. 5

7.COBB, W.S., KERCHER, K.W., HENIFORD, B.T., Surg.Innov., 12, 2005, p. 63

8.MARINESCU, S.A., BEJINARIU, C.G., BADEANA, A., TANASESCUCIUVICA, S., ENESCU, D.M., Rev.Chim.,70, (4), 2019, 1234.

9.HOLSTE, J.L., Int. Surg., 90, 2005, p. 10

10.SOUTO, L.R.M., CARDOSO, L.A.A., CLARO, B.M., Aesth.Plast.Surg., 35, no. 2, 2011, p. 184

11. MARINESCU, S.A., BEJINARIU, C.G., SAPTE, E., MARINAS, M.C., GIUGLEA, C., Rom J Morphol Embryol, 60, no.1, 2019, p. 87

12.MACHLEIDT, A., SCHMIDT-FEUERHEERD, N., BLOHMER, J.U., Arch. Gynecol. Obstet., 298, no. 4, 2018, p. 755

13.MARINESCU, S., BEJINARIU, C.G., GIUGLEA C., Mater.Plast., 56, (1), 2019, 229.

14.ELLIS, H. L., ASAOLU, O., NEBO, KASEM, A., World Journal of Surgical Oncology, 14, 2016, p. 121

15.CELEA, C., DRAGOSLOVEANU, S., POP, M., CELEA, C., Journal of Pediatric Ophtalmology and Strabismus, 53, no. 6, 2016, p. 357

16.BENEDEK, Z., BAUER, O. H., SORLEA, S., COROS, M.F., MEZEI, T., GEORGESCU, R., Mater.Plast., 55, (2), 2018, 196

17.TESSLER, O., REISH, R.G., MAMAN, D.Y., Plast. Reconstr .Surg., 133, 2014, p.90

Manuscript received: 18.11 .2019 